\title{
Monoclonal and polyclonal immunoglobulin G deposits on tubular basement membranes of native and pretransplant kidneys: $A$ retrospective study
}

Anri Sawada ( $\square$ anri-sawada@nms.ac.jp)

Nippon medical school https://orcid.org/0000-0002-0411-7332

Masayoshi Okumi

Tokyo Joshi lka Daigaku

Shigeru Horita

Tokyo Joshi lka Daigaku

Tomomi Tamura

Tokyo Joshi lka Daigaku

Sekiko Taneda

Tokyo Joshi lka Daigaku

Hideki Ishida

Tokyo Joshi lka Daigaku

Motoshi Hattori

Tokyo Joshi lka Daigaku

Kazunari Tanabe

Tokyo Joshi Ika Daigaku

Kosaku Nitta

Tokyo Joshi lka Daigaku

Kazuho Honda

Showa Daigaku

Junki Koike

Sei Marianna Ika Daigaku

Yoji Nagashima

Tokyo Joshi Ika Daigaku

Akira Shimizu

Nihon Ika Daigaku

Research article

Keywords: renal pathology, tubular basement membrane, tubular basement membrane immune deposit, electron-dense deposit, light chain and heavy chain deposition disease 
Posted Date: June 25th, 2020

DOl: https://doi.org/10.21203/rs.3.rs-22486/v1

License: (c) (i) This work is licensed under a Creative Commons Attribution 4.0 International License. Read Full License 


\section{Abstract}

\section{Background}

Monoclonal tubular basement membrane immune deposits (TBMID) are identified by renal biopsy and are associated with progression of interstitial injury in renal allograft. However, the significance of TBMID in the native kidney remains unclear.

\section{Method}

We retrospectively analyzed 3,126 native kidney biopsies and 1,724 zero-hour biopsies performed between 2008 and 2018 in our institution.

\section{Results}

The rate of immunoglobulin G (IgG) TBMID was found to be $5.2 \%$ among native kidney biopsies and 0.4 $\%$ among zero-hour biopsies. The rate of IgG TBMID was relatively common in the case of diabetic nephropathy (31.3\%) and lupus nephritis (25.5\%), but rare in IgA nephropathy (IgAN) (1.1\%). Monoclonal IgG TBMID was identified in seven cases, including three zero-hour biopsies. The pathological diagnosis from native kidney biopsy was IgAN in two cases, antineutrophil cytoplasmic antibody-related vasculitis in one, and Focal segmental glomerulosclerosis in one. Zero-hour biopsy revealed IgAN in one case and mild atherosclerosis in two. The combination of $\lg \mathrm{G} 1 \mathrm{~K}$ was observed in two cases, $\lg \mathrm{g} 1 \lambda$ in three, and IgG2K in two. Interstitial injury is not severe in all cases. Electron microscopy revealed powdery electrondense deposits. Monoclonal gammopathy of undetermined significance was diagnosed in one case with IgA nephropathy. Although One patient developed renal failure, all others exhibited stable renal function.

\section{Conclusion}

Monoclonal IgG TBMID in the native kidney is not associated with renal prognosis or interstitial injury. However, this could be a useful immunopathological parameter for early identification of cases requiring hematological investigation and microscopic evaluation.

\section{Background}

Immunofluorescence staining has been an important tool in renal biopsy to evaluate glomerular immune type deposition. Particularly, the distribution of IgG subclass and monoclonal immunoglobulin deposition in glomeruli are important factors to characterize various glomerular diseases [1] [2] [3]. However, there are few studies on tubular basement membrane immune deposits (TBMID). TBNMID is found in cases of lupus nephritis, membranoproliferative glomerulonephritis, membranous nephropathy, drug-induced tubulointerstitial nephritis, Sjögren's syndrome, immunoglobulin G (IgG) 4-associated nephritis, and other autoimmune diseases [4] [5]. Although previous studies have considered IgG subclass distribution in tubular basement membrane (TBM) for lupus nephritis and IgG4-related diseases, the significances still remain unclear [6] [7]. 
Glomerulonephritis that is characterized by monoclonal IgG deposition has been reported as proliferative glomerulonephritis with monoclonal immunoglobulin deposit (PGNMID) [8]. PGNMID often lacks monoclonal gammopathy and clinical features and pathogenesis of PGNMID are different from other immune-related glomerulonephritis [9]. We have previously reported the association of monoclonal IgG TBMID with the formation of electron-dense deposition (EDD) in the TBM with subsequent progression of interstitial fibrosis and tubular atrophy (IFTA) [10]. However, to the best of our knowledge, there are no reports on monoclonal TBMID in native kidneys or pretransplant kidneys.

In the present study, we analyzed biopsy specimens of polyclonal and monoclonal IgG TBMID in native kidneys and pretransplant kidneys. This retrospective clinicopathological study aimed to determine the clinicopathological features of polyclonal and monoclonal IgG TBMID.

\section{Methods}

We recruited all patients between April 2008 and March 2018 at Tokyo Women's Medical University with data of native kidney and zero-hour kidney biopsy. In a zero-hour biopsy, a cortical wedge is taken immediately after revascularization on completion of vascular anastomosis. All renal biopsies were examined with standard light microscopy (LM), immunofluorescence (IF) and transmitted electron microscopy (EM). For LM, all biopsies were routinely stained with hematoxylin and eosin, periodic acidSchiff, Masson's trichrome, and PAS methenamine silver. For IF, 2- $\mu \mathrm{m}$ cryostat sections were dried and stained with fluorescein isothiocyanate (FITC)-conjugated polyclonal antibodies against IgG; IgA; IgM; complement components $3,1 \mathrm{q}$, and $4 \mathrm{~d}$ (C3c, C1q, and C4d); fibrinogen; $\mathrm{K}$ light chain; and $\lambda$ light chain (Dako Corp., Carpinteria, CA, USA). Measurement of IgG was performed on 2- $\mu \mathrm{m}$ cryostat sections stained with FITC-conjugated monoclonal antibodies against IgG1, IgG2, IgG3, and IgG4 (The Binding Site Inc., San Diego, CA, USA). Monoclonal IgG TBMID was identified by the presence of monoclonal staining for a single light-chain isotype and a single-heavy chain subclass in TBM samples. All biopsies were reviewed by three independent pathologists. Patient information was collected from medical records. This study and all its protocols were approved by the ethics committee at Tokyo Women`s Medical University (No. $5415)$.

\section{Results}

\section{Distribution of IgG subclasses in the tubular basement membrane}

We analyzed 3,126 native renal and 1,724 zero-hour biopsy specimens in the present study. Table 1 presents the IgG subclass distribution of TBMID in relation to various diseases. We identified IgG TBMID in $164(5.2 \%)$ patients who underwent native renal biopsy and six $(0.4 \%)$ who underwent zero-hour biopsy. The rate of TBMID is diabetic kidney disease (31.3\%), lupus nephritis (25.5\%), membranous nephropathy (14.9\%), IgA nephropathy (1.1\%), membranoproliferative glomerulonephritis (14.0\%), ANCArelated crescentic glomerulonephritis (9.3\%), light and heavychain deposition disease (100\%), focal segmental glomerulosclerosis (7.7\%) and IgG4-related disease (37.5\%). The rate of IgG1 deposition (92.5 
\%) was significantly higher than the other subclasses (IgG2 [66.0\%], IgG3 [56.4 \%], and IgG4 [53.2 \%]). In lupus nephritis, 17 of 38 cases that showed TBM deposits showed positive staining for all four subclasses. The rate of IgG3 and IgG4 deposition was lower than the other three IgG subclasses. In IgG4related disease, all cases were positive for all four subclasses. In zero-hour biopsies, TBMID was very rare $(0.35 \%)$, and all cases showed deposition of monotypic IgG subclass in the TBM.

\section{Pathological findings of monoclonal immunoglobulin $\mathrm{G}$ tubular basement membrane immune deposits}

Pathological characteristics of monoclonal IgG TBMID are presented in Table 2. Monoclonal IgG TBMIDs were identified in seven patients, including three who underwent 0-hour biopsy. On the other hand, neither monoclonal IgA nor IgM TBMID were identified. Pathological diagnoses were IgA nephropathy $(n=3$, including one patient who underwent zero-hour biopsy), mild arteriosclerosis $(n=2)$, antineutrophil cytoplasmic antibody-related vasculitis $(n=1)$, and focal segmental glomerulosclerosis (FSGS) $(n=1)$. Other two zero-hour biopsies showed only mild atherosclerosis. Neither tubulointerstitial nephritis nor cast in tubules were found in any cases. The median IFTA was 5\% (range: $0 \%-30 \%$ ). Glomerular IgG deposition was found to be negative in all cases by IF analysis. Monoclonal IgG deposition was present in the proximal tubule in all cases. The combinations of IgG subclass and light chain in TBM were IgG1 $\mathrm{K}$ $(n=2), \lg G 1 \lambda(n=3)$, and $\lg G 2 K(n=2)$. The IF staining pattern was linear, and C3c, C4d, and C1q staining was negative in the TBM in all cases (Figures 1,2, and 3). EM analysis revealed the powdery EDD in all cases (Figure 4). Only one case showed slight EDD in the glomerulus (case 1). Therefore, FSGS lesion was considered a secondary FSGS caused by light chain and heavy chain deposition. On the other hand, other cases did not show the powdery EDD in glomerulus.

\section{Characteristics of patients with monoclonal immunoglobulin $\mathbf{G}$ tubular basement membrane immune deposits}

Table 3 presents the clinical characteristics of patients with monoclonal IgG TBMID including 4 patients performed of native kidney biopsy and three donor patients of zero-hour biopsy (4 male and 3 female). The median age at transplantation was $53(21-69)$ years and median serum creatinine at the time of biopsy was $0.81(0.56-1.4) \mathrm{mg} / \mathrm{dL}$. Median proteinuria at biopsy was 0.2 (negative-3.0) g/day. Hepatitis $\mathrm{B}$ or $\mathrm{C}$ infection or other infectious disease was not identified. Monoclonal gammopathy of undetermined significance was diagnosed in one patient (case 6) based on bone marrow analysis. No other patients had circulating paraprotein or hematologic disease. All four patients who underwent native kidney biopsy received treatment for glomerular disease. one patient received rituximab treatment (case 1), while three received steroid treatment for IgA nephropathy and ANCA-associated vasculitis. Three donor patients received no treatment. All three cases of zero-hour biopsy were observed without treatment. The median follow-up period from biopsy was $3.5(0.5-6)$ years. Although one patient progressed to end-stage renal disease (ESRD) 6 years after biopsy despite rituximab treatment (case 1), all other patients exhibited stable renal function. Median serum creatinine at the last follow-up was $0.8(0.57-1.19) \mathrm{mg} / \mathrm{dL}$. 


\section{Discussion}

In this study, we examined the distribution of IgG subclass in TBM in various stages of renal disease. Additionally, we found seven cases of monoclonal IgG TBNMID and examined their clinicopathological characteristics.

In humans, there are four subclasses of $\operatorname{lgG}(\operatorname{lgG} 1-4)$, which are present in different amounts in the circulation; the proportions of $\operatorname{lgG} 1,2,3$, and 4 being $60.3 \%-71.5 \%, 19.4-31.0 \%, 5.0 \%-8.4 \%$, and $0.7 \%-$ $4.2 \%$, respectively [11]. Each IgG subclass is associated with particular immunological and functional roles. In glomerular disease such as membranous nephropathy, the specific deposition of IgG subclasses in the glomerulus has diagnostic significance [12]. The roles of IgG1 and IgG3 are to activate the complement system, whereas IgG2 and IgG4 have little involvement with this activation [13]. The present study found TBMID to be common in the context of diabetic nephropathy, membranous glomerulonephritis, and lupus nephritis, with the rate of IgG1 deposition being much higher than other IgG subclasses. However, no differences were observed between the other three IgG subclasses. About half of the cases of lupus nephritis showed deposition of all subclasses, with $\lg \mathrm{G} 1$, $\lg \mathrm{G} 2$, and $\lg \mathrm{G} 3$ being predominant. These findings are consistent with prior studies on IgG subclass deposition in the glomeruli [14]. On the other hand, TBMID is very rare in IgA nephropathy and living transplant donors. All these cases showed monotypic IgG deposition in TBM. This suggests that examination of IgG subclass in the TBM should be carried out for such cases.

In the seven cases of monoclonal TBMID that were found in the present study, IgG1 was the most common subclass, and the ultrastructure of the EDD in the TBM exhibited a powdery structure. In contrast, PGNMID is characterized by non-organized EDD and the most common subclass is IgG3 [9]. We have previously reported the presence of monoclonal IgG TBMID in renal allograft, and found monoclonal IgG1K TBMID to exhibit non-organized EDD formation in the TBM and to be associated with the progression of IFTA [10]. However, the present study did not identify remarkable IFTA in any case. Taken together, our findings suggest that monoclonal IgG TBMID in native kidney occurs via a different mechanism than in the case of PGNMID and IgG TBMID in renal allograft. This may be because the pathogenesis of TBM immune complex formation is different from that in the glomeruli and renal allograft [6] [15]. The immune complex in TBM is presumed to form in situ with DNA or DNA-binding proteins such as previously deposited or endogenous tubular epithelial proteins [6]. Furthermore, various immunosuppressants or molecular-targeted drugs are used during renal allograft to suppress rejection or treat de novo or recurrent glomerulonephritis; eculizumab has been reported to bind to and deposit IgG in the TBM, resulting in TBMID [16]. Although the mechanism of this pathogenesis remains unclear, it is possible that the change of humoral immunity due to transplantation or drug interactions contribute to pathological changes.

The pathological findings of the present study reflect LHCDD; the most common glomerular manifestation of LCDD is nodular glomerulosclerosis, while many cases display normal glomeruli in LM. Moreover, some cases of LCDD exhibit deposits localized to the renal tubules [17]. There are some 
differences between the cases presented here, however; while LHCDD and HCDD are often characterized by deposition of $\mathrm{C} 3 \mathrm{c}$ and $\mathrm{C} 1 \mathrm{q}$ [18], we did not observe such deposition in the TBM. Moreover, while LHCDD is well known for its poor prognosis, almost all our cases had good renal and life prognoses. The pathological significance of these differences is unclear. Previous reports have demonstrated that the various subtypes of Bence-Jones protein have different roles in the kidney [19], which suggests that pathological and clinical changes depend on the type of free light and heavy chain. The molecular characteristics of light and heavy chains that lead to the different morphological patterns of tissue deposition; such as casts, granules, crystals, and fibrils; have not yet been clarified, although the physicochemical properties of the individual light chain or local factors in the various renal compartments are thought to be responsible [20]. Further investigation is required to clarify the pathogenesis, relevance to treatment response, and prognosis.

In this study, one patient progressed to ESRD, which was diagnosed as FSGS caused by light chain and heavy chain deposition and showed nephrotic-range proteinuria and relatively high serum creatinine at the time of renal biopsy. Some reports have stated that renal insufficiency is an independent risk factor for MIDD [21] [22]. These results suggest that early detection of renal dysfunction and comprehensive histologic evaluation, including IF and EM, is important in monoclonal IgG TBMID.

There are several limitations of this study which should be acknowledge. First, due to the retrospective nature, some laboratory data were not obtainable. Analysis of paraprotein by IF, immune electrophoresis, or kappa/lambda ratio measurement were not carried out in all cases. Second, monoclonal IgG and lightchain deposition was only evaluated by IF. The affinity of IgG to the TBM varies depending on the type of immunoglobulin and light chain. Although each IgG subclass except IgG4 and both $\mathrm{k}$ 冈and $\lambda$ light chain were detected, we cannot deny that the affinity of immunoglobulin and light chain for the TBM may have affected our results. Third, IgG subclass was not examined in all cases. In our laboratory, IgG subclass staining is not routinely performed; for example, IgG subclass staining is carried out to distinguish between idiopathic or secondary membranous nephropathy. However, it is known that there many nonspecific IgG deposits occur on the TBM in diabetic nephropathy. Thus, many cases did not undergo IgG subclass staining and there may be more cases of monoclonal IgG TBMID than our results suggest. Moreover, renal diseases that are complicated with monoclonal IgG TBMID may be more diverse.

\section{Conclusions}

In conclusion, IgG TBMID in the native kidneys is associated with formation of EDD on the TBM but does not influence renal prognosis or IFTA progression. This is different to findings observed in renal allograft. However, IgG TBMID might be a potentially useful immunopathologic parameter for cases of MIDD lacking monoclonal glomerular IgG deposition or where there is asymptomatic underlying hematologic disorder. Because such cases could easily progress to renal failure, IgG TBMID may represent an important biomarker. Careful ultrastructural and immunohistochemical analysis of the type of deposit on the TBM as well as hematological investigations could be beneficial in routine clinical practice. 


\section{Tables}

Table.1 IgG subclass distribution of TBMD in various disease

\begin{tabular}{|c|c|c|c|c|c|c|c|c|}
\hline & Tetal & $\begin{array}{l}\text { log depesits } \\
\text { in TEN }\end{array}$ & $\begin{array}{l}\text { Propostion of ligh } \\
\text { depenit in TBu } \\
\text { (S) }\end{array}$ & $\begin{array}{l}\text { Cases is which bG } \\
\text { netelans mas ebtained }\end{array}$ & $\lg 91$ & la & ngas & log \\
\hline total & & & & 94 & * & 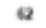 & so & st \\
\hline Diabesc Uidney drease & os & sa & 34.3 & 4 & 4 & 3 & 1 & 2 \\
\hline IgA nephtrogatiy & 1157 & o & 3 & 3 & 3 & 0 & 0 & - \\
\hline Lupun septuikst & 16 & a & 25s & as & $x$ & $n$ & 32 & $n$ \\
\hline Mumbranows neptropally & set & $n$ & 149 & 21 & $x$ & 13 & $\pi$ & ss \\
\hline MecN & 0 & 6 & 960 & 5 & s & 3 & 4 & 4 \\
\hline Esas & is & s & $m$ & 4 & 4 & 2 & 3 & 4 \\
\hline ANCA relaned crescentic GN & $\omega$ & $\mathbf{n}$ & 93 & $\boldsymbol{r}$ & 6 & 2 & 3 & 1 \\
\hline L4CDO & 5 & s & $w 0$ & 5 & 4 & 1 & 0 & ? \\
\hline IgCA-related TIN & - & 3 & 37. & 3 & 3 & 3 & 3 & 3 \\
\hline TN & $\boldsymbol{v}$ & 1 & $u$ & 1 & 1 & 0 & 0 & $\bullet$ \\
\hline Atterith & 2 & 1 & $s$ & 1 & 1 & 0 & 0 & 1 \\
\hline 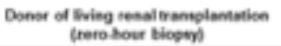 & $m$ & 6 & as & 5 & 2 & 3 & 0 & - \\
\hline
\end{tabular}

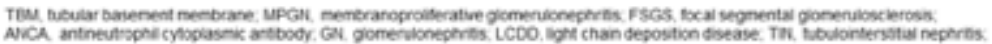

\begin{tabular}{|c|c|c|c|c|c|c|c|c|c|c|c|}
\hline no & Ansery & Daphonis & TIA & 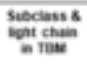 & $\begin{array}{l}\text { Depesincen } \\
\text { Bomet }\end{array}$ & $\begin{array}{c}\text { Wonscied } \\
\text { y6 } \\
\text { in }\end{array}$ & 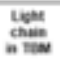 & $\begin{array}{l}c x \\
\min \\
\min \end{array}$ & $\begin{array}{l}a \\
\text { i⿱ } \\
\text { na }\end{array}$ & $\begin{array}{l}\mathrm{Cla}_{4} \\
\min \end{array}$ & Depent \\
\hline 1 & Nateve vitnery & Inss & 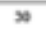 & weth & these & 2 & 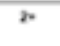 & 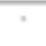 &. &. &. \\
\hline 2 & Nabeve vitnery & max & 5 & 105to & Lineer & - & - & \pm & . & . & 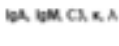 \\
\hline , & $\begin{array}{c}\text { Nobeve vetery } \\
\text { bespey }\end{array}$ & wex & 5 & wots & Unes & - & 2 & . & - & . & 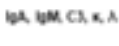 \\
\hline 4 & Nobeventry & $\begin{array}{l}\text { Asca mascinter } \\
\text { nacivine }\end{array}$ & $n$ & $\operatorname{los} x$ & Lueser & $2=$ & 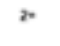 & . & & . & wax $x$ \\
\hline 5 & Zeno-mour ineng & anenoschersis & - & $\log x$ & Lineer & - & - & . & & . & \\
\hline 6 & 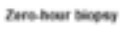 & 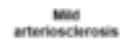 & 5 & $\operatorname{loct} x$ & Lineer & * & 20 & . & . & . & . \\
\hline , & zere hour besey & eas & $n$ & $\log x$ & times & . & . & . & . & . & lox cas \\
\hline
\end{tabular}

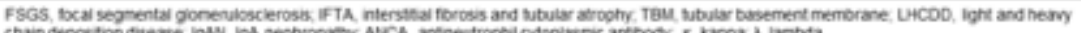


Table.3 Patient characteristics of the patients with monoclonal lgG deposits in TBM

\begin{tabular}{|c|c|c|c|c|c|c|c|c|}
\hline 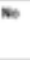 & at beopery & 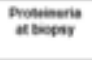 & Menateris & $\begin{array}{l}\text { Inbence of } \\
\text { oropisenemis }\end{array}$ & 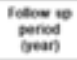 & 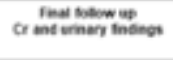 & Mestment & conplication \\
\hline , & 4 & 3 & . & Negative & 6 & HO & คTX & hoes of androme \\
\hline 2 & 0.74 & 6 & so easpet & Negation & is & 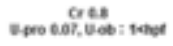 & $\mathrm{nel}$ & neese \\
\hline 3 & ose & 02 & somape & Negantere & 1 & 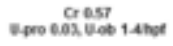 & $\begin{array}{c}\text { Toentivectiomy } \\
\text { mork }\end{array}$ & $\begin{array}{l}\text { chroenc Thyoustis } \\
\text { faciai parsalvis }\end{array}$ \\
\hline 4 & asr & es & somape & Unanimen & 4 & 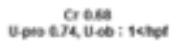 & neu & 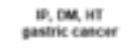 \\
\hline $\mathrm{s}$ & ano & Nentroe & ethet & Negative & , & 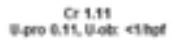 & None & $\mathrm{Pa}$, ит, нL \\
\hline 6 & eas & sesputre & sthet & yox & as & 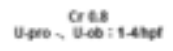 & None & Whet \\
\hline 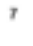 & ass & Sentive & etter & Nesative & 4 & 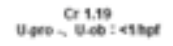 & Sones & None \\
\hline
\end{tabular}

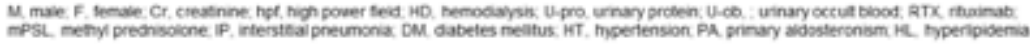

\section{Abbreviations}

TBMID: tubular basement membrane immune deposits, IgG: immunoglobulin G, IgAN: IgA nephropathy, TBM: tubular basement membrane, PGNMID: proliferative glomerulonephritis with monoclonal immunoglobulin deposit, EDD: electron-dense deposition, IFTA: interstitial fibrosis and tubular atrophy, LM: light microscopy, IF: immunofluorescence, FITC: fluorescein isothiocyanate, C3: complement components 3, C1q: component 1q, C4d: component 4d, EM: electron microscopy, FSGS: focal segmental glomerulosclerosis

\section{Declarations}

\section{Authors' contributions}

A.S designed the research and wrote manuscript. ST, $\mathrm{KH}$, and $\mathrm{JK}$ supported in writing this report and revised it. SH, AS, YN analyzed pathological information. MO, TT, HI, KT MH, and $\mathrm{KN}$ analyzed clinical information. All authors read and approved the final manuscript.

\section{Acknowledgments}

We thank Hideki Nakayama and Mayuko Ohno for their technical support and Jun Ino, Sachiko Wakai, Takashi Naito, Kazuya Omoto and Hiroki Shirakawa for the support of collecting medical information. The authors would like to thank Enago (http://www.enago.jp) for the English language review.

\section{Funding}

The authors received no specific funding for this work.

\section{Availability of data and materials}


The datasets used and/or analyzed during the current study are available from the corresponding author on reasonable request.

\section{Ethics approval and consent to participate}

The study was carried out in compliance with the Declaration of Helsinki. Informed consent was obtained by optout as it was a retrospective study. This study and all its protocols were approved by the ethics committee at Tokyo Women`s Medical University (No. 5415).

\section{Consent for publication}

Consent for the publication was obtained by optout in line with the local ethics committee's. The documents regarding optout are available from the corresponding author on reasonable request.

\section{Competing interests}

The authors declare that they have no competing interests.

\section{References}

1. Safar-Boueri L, Piya A, Beck LH, Jr., Ayalon R. Membranous nephropathy: diagnosis, treatment, and monitoring in the post-PLA2R era. Pediatr Nephrol 2019; 1-12

2. Almaani S, Meara A, Rovin BH: Update on lupus nephritis. Clin J Am Soc Nephrol 2017; 12: 825-835

3. Motwani SS, Herlitz L, Monga D, Jhaveri KD, Lam AQ. Paraprotein-related kidney disease: glomerular diseases associated with paraproteinemias. Clin J Am Soc Nephro/ 2016; 11: 2260-2272

4. Park MH, D'Agati V, Appel GB, Pirani CL. Tubulointerstitial disease in lupus nephritis: relationship to immune deposits, interstitial inflammation, glomerular changes, renal function, and prognosis. Nephron 1986; 44: -309-319

5. Bracamonte E, Leca N, Smith KD, et al. Tubular basement membrane immune deposits in association with BK polyomavirus nephropathy. Am J Transplant 2007; 7: 1552-1560

6. Satoskar AA, Brodsky SV, Nadasdy G, et al. Discrepancies in glomerular and tubulointerstitial/vascular immune complex IgG subclasses in lupus nephritis. Lupus 2011; 20: 13961403

7. Hemminger J, Nadasdy G, Satoskar A, Brodsky SV, Nadasdy T. IgG subclass staining in routine enal biopsy material. The American journal of surgical pathology 2016; 40: 617-626

8. Nasr SH, Markowitz GS, Stokes MB, et al. Proliferative glomerulonephritis with monoclonal lgG deposits: a distinct entity mimicking immune-complex glomerulonephritis. Kidney Int 2004; 65: 85-96

9. Nasr SH, Satoskar A, Markowitz GS, et al. Proliferative glomerulonephritis with monoclonal IgG deposits. J Am Soc Nephrol 2009; 20: 2055-2064

10. Sawada A, Kawanishi K, Horita S, et al. Monoclonal immunoglobulin G deposits on tubular basement membrane in renal allograft: is this significant for chronic allograft injury? Nephrol Dial Transplant 
2018; 711-717

11. Hamilton RG. Human IgG subclass measurements in the clinical laboratory. Clin Chem 1987; 33 : 1707-1725

12. von Haxthausen F, Reinhard L, Pinnschmidt HO, et al. Antigen-specific IgG subclasses in primary and malignancy-associated membranous nephropathy. Front Immunol 2018; 9: 3035

13. Burton DR, Woof JM. Human antibody effector function. Adv Immunol 1992; 51: 1-84

14. Kuroki A, Shibata T, Honda H, Totsuka D, Kobayashi K, Sugisaki T. Glomerular and serum IgG subclasses in diffuse proliferative lupus nephritis, membranous lupus nephritis, and idiopathic membranous nephropathy. Internal Medicine (Tokyo, Japan) 2002; 41: 936-942

15. Nangaku M, Couser WG. Mechanisms of immune-deposit formation and the mediation of immune renal injury. Clin Exp Nephrol 2005; 9: 183-191

16. Herlitz LC, Bomback AS, Markowitz GS, et al. Pathology after eculizumab in dense deposit disease and C3 GN. J Am Soc Nephrol 2012, 23: 1229-1237

17. Gokden N, Barlogie B, Liapis H. Morphologic heterogeneity of renal light-chain deposition disease. Ultrastruct Pathol 2008; 32: 17-24

18. Hogan JJ, Alexander MP, Leung N. Dysproteinemia and the Kidney: Core Curriculum 2019. Am J Kidney Dis 2019.

19. Gallo G. Renal complications of B-cell dyscrasias. N Engl J Med 1991; 324: 1889-1890

20. Gokden N, Cetin N, Colakoglu N, et al. Morphologic manifestations of combined light-chain deposition disease and light-chain cast nephropathy. Ultrastruct Patho/ 2007; 31: 141-149

21. Gerth J, Sigusch H, Illner N, et al. Renal manifestations of light chain associated diseases epidemiology and prognosis. Dtsch Med Wochenschr 2013; 138: 305-312

22. Kourelis TV, Nasr SH, Dispenzieri A, et al. Outcomes of patients with renal monoclonal immunoglobulin deposition disease. Am J Hematol 2016; 91: 1123-1128

\section{Figures}




\section{Case1}

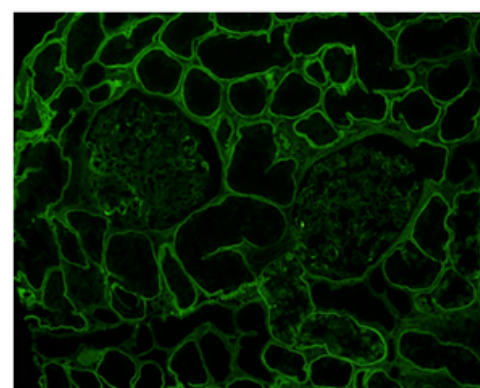

IgG1
IgG2

IgG3

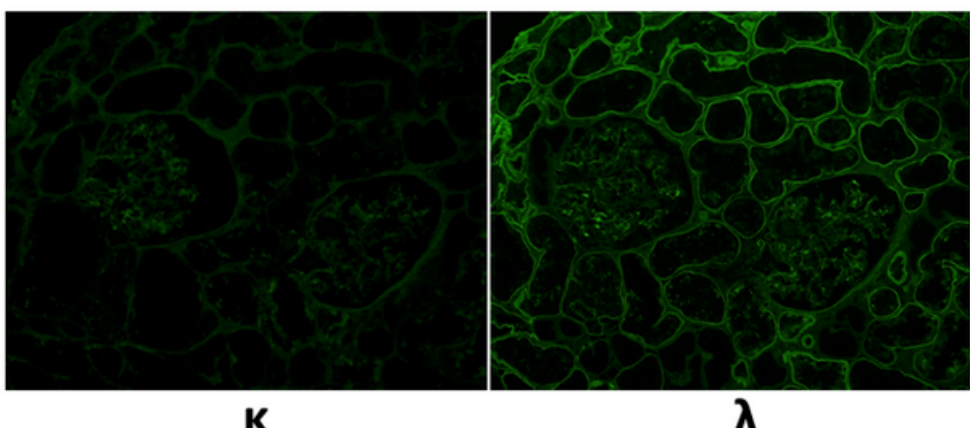

IgG4

\section{Figure 1}

Light microscopy images of immunofluorescence analysis of allograft biopsy (case 1). Linear immunoglobulin G1 (IgG1) and light chain $\lambda$ deposition was identified in the tubular basement membrane. Staining for $\lg \mathrm{G} 2$, IgG3, IgG4, IgA, IgM, and light chain $\mathrm{k}$ was negative.

\section{Case3}

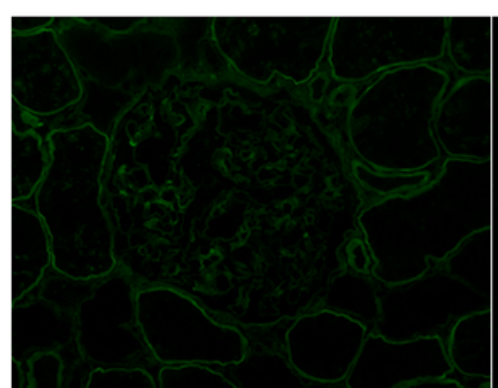

IgG1

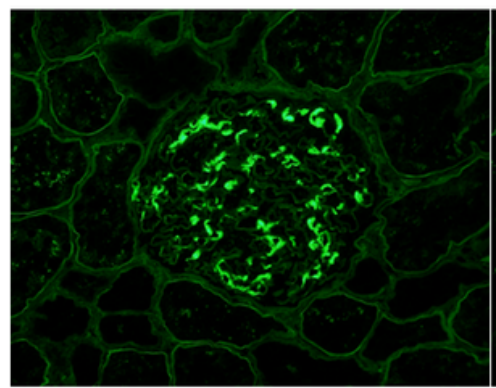

K
IgG2

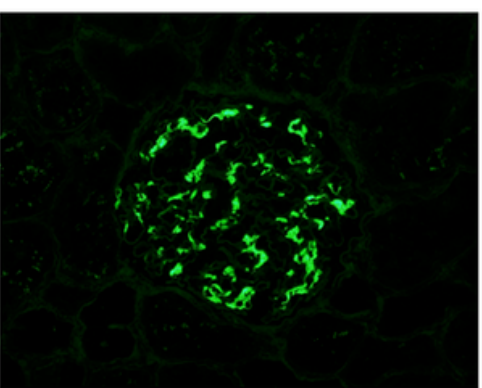

$\lambda$

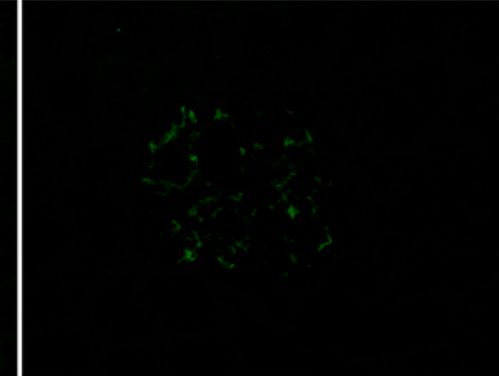

IgG3

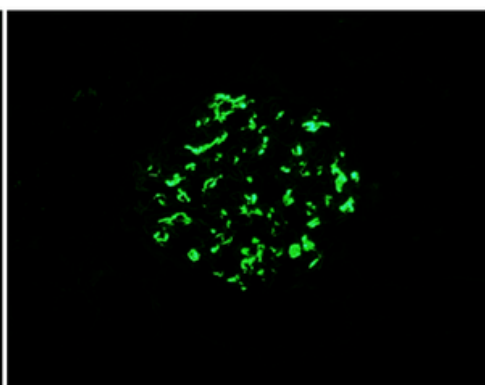

$\lg A$

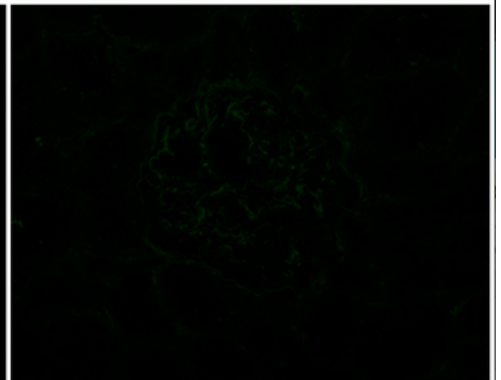

IgG4 


\section{Figure 2}

Light microscopy images of immunofluorescence analysis of allograft biopsy (case 3). Linear immunoglobulin G1 (IgG1) and light chain $\mathrm{K}$ deposition was observed in the tubular basement membrane. Staining for $\lg G 2$, $\lg G 3$, $\lg G 4$, $\lg A$, $\lg M$, and light chain $\lambda$ was negative, while $\lg A, C 3$, and light chain $\mathrm{K}$ and $\lambda$ were detected in the glomeruli.

\section{Case5}

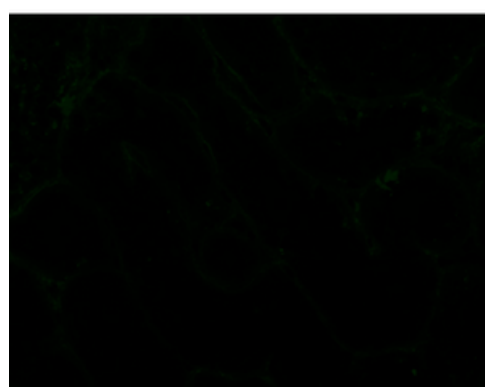

$\operatorname{lgG1}$

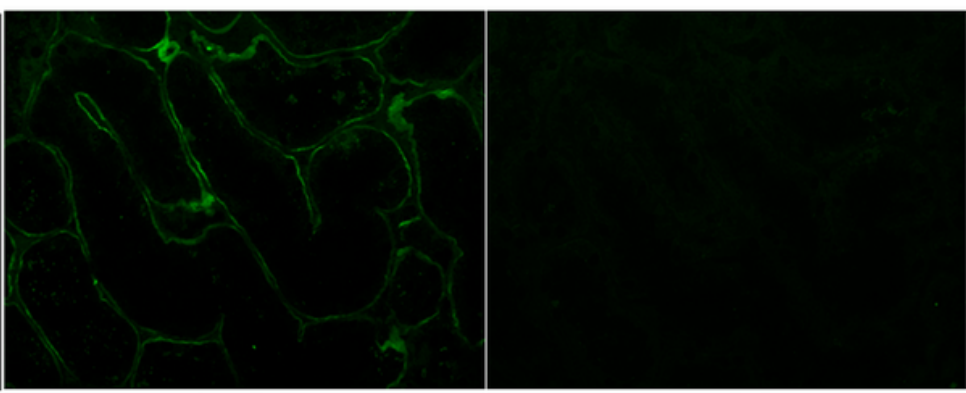

IgG2

IgG3

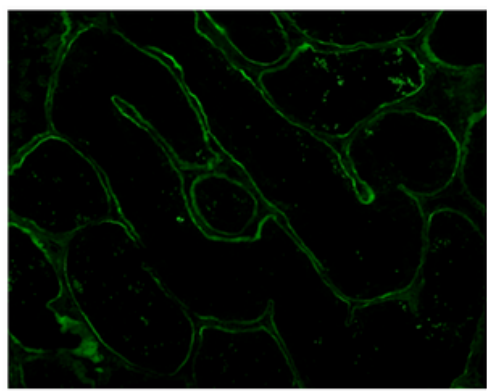

$\mathbf{K}$

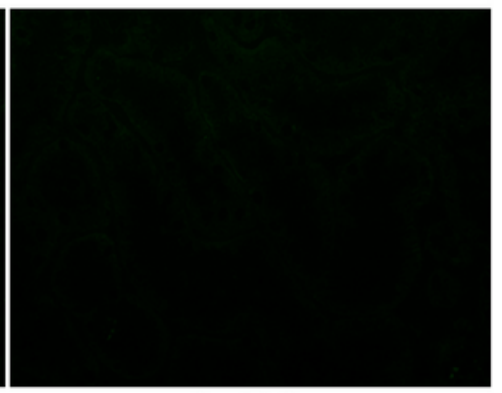

IgG4

\section{Figure 3}

Light microscopy images of immunofluorescence analysis of allograft biopsy (case 5). Linear immunoglobulin $\mathrm{G} 2$ ( $\operatorname{lgG} 2$ ) and light chain $\mathrm{K}$ deposition was observed in the tubular basement membrane. Staining for $\lg \mathrm{G} 1$, IgG3, $\lg G 4$, $\lg \mathrm{A}$, IgM, and light chain $\mathrm{K}$ were negative. 
Case1

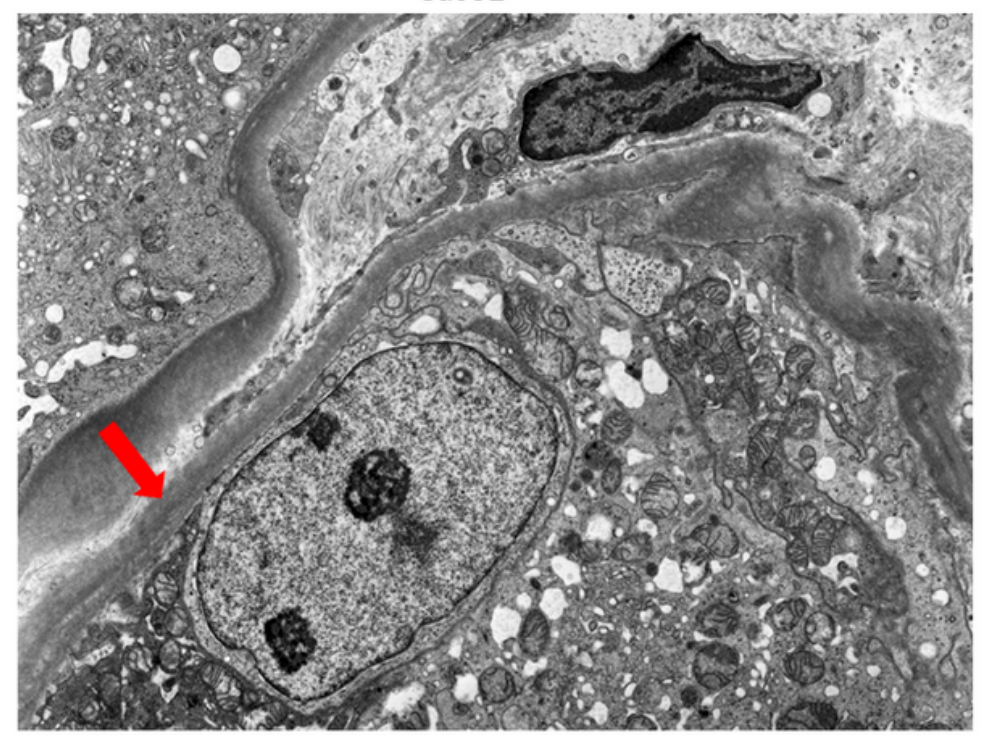

Case2

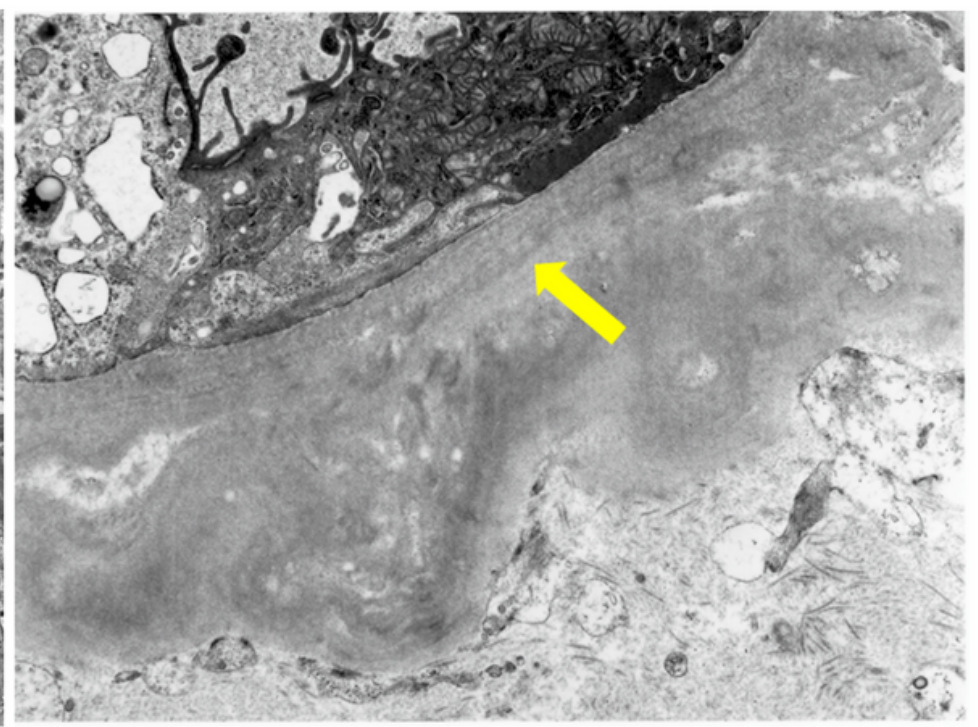

\section{Figure 4}

4 Electron microscopy images of renal biopsies. Powdery electron-dense deposits (indicated by the red and yellow arrows) were observed in the tubular basement membrane of cases 1 (at $4000 \times$ magnification) and 2 (at $7000 \times$ magnification) 